\title{
A Glance into Virtual Reality Development Using Unity
}

\author{
Cosmina ISAR \\ The Bucharest University of Economic Studies, Romania \\ isarcosmina@gmail.com
}

\begin{abstract}
Coming from the $2 D$ and $3 D$ games that everybody is very familiar with, these days the world has been taken by storm with the introduction of virtual reality technology. In this paper I covered the essence of this new empowering technology through things like: a short representative definition and classification, the hardware requirements for stepping into the VR world, and the most important aspect which is the applicability of this technology in the society that we live in. Also, the main purpose of this paper was to identify which VR game engine a beginner should be using for their first project. Through the research that has been done in this article, I come to the conclusion that the most suitable game engine in this case is Unity. To further prove my statement, I developed a small project that consists of a $3 D$ VR maze which shows how easy it is to learn and develop using Unity.
\end{abstract}

Keywords: Virtual reality, Unity, Game engine

1 Introduction

Nowadays, virtual reality is known as the technology that allows for replacing the real world by a synthetic one, inducing users the feeling of being in another world [1]. The most representative type of application that truly makes someone feel that way, is known as a VR game. Thus, virtual reality and digital games are two area which share many similar characteristics among which the most important are: both of them exploit the technological breakthroughs of several fields like electronics, computer power etc., and also, they focus on creating the best possible realistic 3D environments [2].

My contribution on this paper is to help someone who has never developed a VR game choose the best game engine and, at the same time, because of the higher demand in this technology, to widespread the interest in this area. Furthermore, to prove the easiness of developing in virtual reality I will describe, based on an example, the steps necessary for making a simple VR game.

\section{Overview of virtual reality}

Virtual reality (VR), also known as virtuality [3], is a technology that involves experiencing various things through our computers, things that don't actually exist. In other words, virtual reality also represents a believable, interactive $3 \mathrm{D}$ computer generated world in which people can explore and interact, thus making them feel like they are there, both physically and mentally.

In other words, virtuality represents a world that is:

- Computer-generated - this part of the equation represents a very important factor in generating a 3D world, because only powerful enough computers can make the experience more immersive and believable;

- Believable - for the VR experience to be induced, users really need to feel like they are in that generated world, so the illusion of virtual reality will fade to back;

- Interactive - to mimic the reality that we live in, users of virtual reality should be able to interact with the elements that are present in the computer-generated world, so the experience gets fulfilled;

- Immersive - to assure a great connection between believable and interaction, virtual reality has to engage both body and mind of users. Even though VR promises a very realistic alternative to our real world, in the current state it faces some limitations like: smell, fell and taste;

- Explorable - as we can explore the surroundings in our world, the VR should give us the same level of freedom. In other words, the VR scene should embrace the same level of complexity and detail as the real world. 
At the same time, there are several types of virtual reality:

- Fully immersive - Three main things are necessary for a completely VR experience. First, a computer-generated world that is highly detailed and complex. Second, a powerful computer that knows where we are heading and who is capable of adjusting the experience accordingly. Third, hardware accessories like head-mounted display, stereo sound and sensory gloves which virtually creates the virtual world.

- Non-immersive - For example, a highly realistic driving simulator on a PC could be classified as non-immersive virtual reality, particularly if it uses peripherals like: a wide screen, surround sound headphones and a realistic wheel.

- Collaborative - Games like Minecraft and Second Life who are about "virtual world" do not count as virtual reality, because they only meet the following criteria: computergenerated, believable, interactive and explorable. Besides all of this, a complete VR experience should be fulfilled by a strong collaboration between players, which represents an important fact that virtual reality tends to lean in the future.

- Web-based - In the late 1980s and early 1990 s, virtual reality was one of the fastestgrowing technology, but the popularity of World Wide Web has minimized the interest in VR. Even though computer scientists developed a way of building virtual worlds on the Web, the vast majority of people were much more interested in the way the Web gave them new ways to access real reality like: new ways to find and publish information and share ideas and experiences with friends through social media [4]. With Facebook's growing interest in the technology, the future of VR seems likely to be both Web-based and collaborative.

- Augmented reality - This technology represents a combination between virtual reality and real world. Basically, AR means adding holograms into the real world which the user can interact with. All of this is possible by using spatial tracking sensors which move the holograms according to the user's movement.

\section{Necessary hardware for virtual reality}

For everybody that is willing to step into the world of virtual reality, a specific set of hardware accessories is needed to made it possible. The set mentioned above might consists of:

- Head-mounted displays that consists of two small screens, one for each eye, a material that is used to stop the light that is coming from the real world and a pair of stereo headphones with the role to give users the awareness of surroundings.

- Immersive rooms which represents an alternative for head-mounted displays, consists of areas that contains special projectors which turn the walls into displays. This highly advanced room also contains a set of specialized sensors who can track people that are inside, thus moving the projected images according to their movement.

- Data-gloves that are used for giving people the ability to interact with the virtual objects making the experience more lifelike. This technology involves strapping highly sophisticated sensors on to ordinary gloves.

\section{Uses of virtual reality}

Being able to simulate real world scenarios, we can say that virtual reality has a very wide range of uses, from education to Medicine.

\subsection{Education}

Learning to pilot a plane, for example, can be a very dangerous activity for an untrained person. But in the last few years, because the VR technology had become better, more and more academies started to implement virtual reality simulators for this activity, and thus making the process of learning to fly safer. And also, because there are no lives at risk, students can learn complicated maneuvers faster.

\subsection{Science}

VR can help scientists visualize their work in a natural way free of any tools like microscopes, computer screens. It also helps them make rapid prototyping, because they can see the final result being placed in the real world 
through the use of augmented reality technology. The first project of this type was called "GROPE" and it was introduced in 1960s at the University of North Carolina and it was about exploring the interactions between protein molecules and drugs [4].

\subsection{Games and entertainment}

When it comes to gaming and entertainment, VR has become more widespread than the other categories mentioned above. At the same time, in these domains, virtual reality is rapidly evolving compared to the others, mainly because there is a higher demand and also because these types of applications are easier to develop.

\subsection{Medicine}

Since 2009 with the introduction of daVinci surgical robot, VR has extended it's uses to medical world. This robot is basically a pair of four arms that are controlled by a surgeon remotely through special VR equipment. This make it possible for other surgeons situated in different places to join in a more complex operation and collaborate together to resolve the case.

\section{Virtual reality in gaming}

In the past few years, because of the higher demand for VR games, more and more companies started to invest in developing specific gadgets for this technology. Right now, in the VR game industry there are two main competitors Oculus Rift and HTC Vive. For a visual representation of them see Figure 1 and Figure 2.

The Oculus Rift is a virtual reality headset developed and manufactured by Oculus VR which is a division of Facebook Inc. It was released on March 28, 2016. This device has a Pentile OLED display with the resolution of $1080 \times 1200$ for each eye, a refresh rate of 90 $\mathrm{Hz}$ and a field of view of 110 degrees. At the same time, it has a pair of audio headphones which are tuned to provide a 3D audio effect. Oculus Rift also has a positioning tracking system and two ergonomically designed controllers [5].
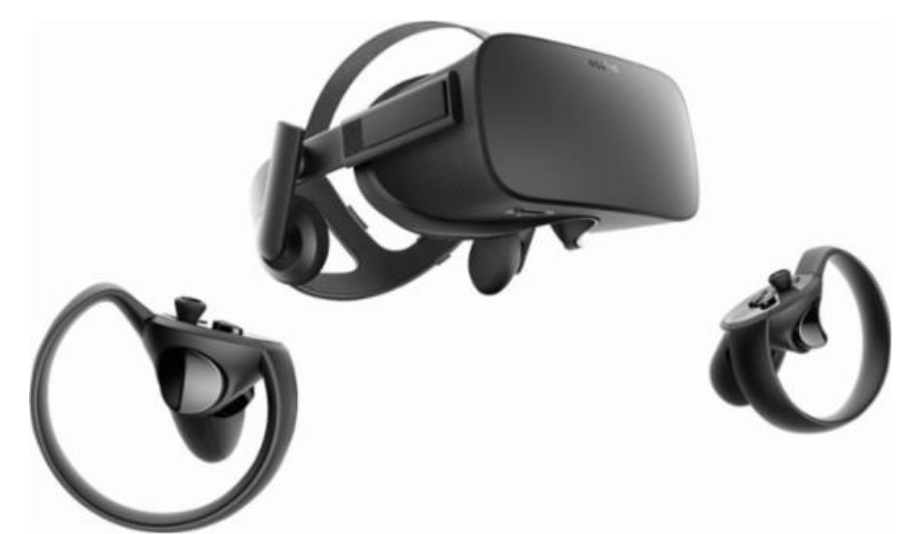

Fig. 1. Oculus Rift headset (source: https://www.bestbuy.com/site/oculus-rift-touch-virtualreality-headset-bundle-for-compatible-windows-pcs-black/5989502.p?skuId=5989502 )

The HTC Vive is also a VR headset developed by HTC and Valve Corporation and it was released on April 5, 2016. It has almost the same configuration of hardware accessories as the
Oculus Rift with the only different factor being the ability to allow users to move in a larger area [6]. 


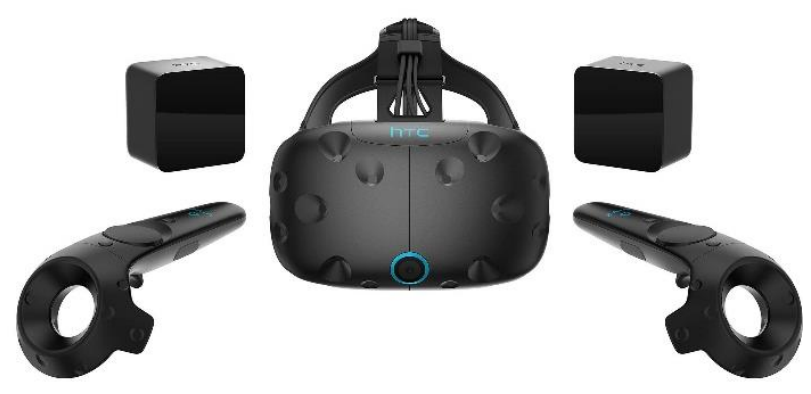

Fig. 2. HTC Vive headset (source: https://www.bhphotovideo.com/c/product/1337110REG/htc_99haln002_00_vive_vr_system.html )

Using all this technology some major companies have already released a great collection of VR games, among which:

- Oculus Rift: Eve: Valkyrie, Lone Echo, Wilson's Heart, The Unspoken, Feral Rites, etc.

- HTC Vive: Arizona Sunshine, Superhot, Fallout 4, L.A. Noire, Project Cars, The Gallery, etc.

\section{VR games development}

To develop a VR ready game, a developer should take into consideration one of the following game engines that fully support this type of technology: Unity, Unreal Engine, CryEngine, AppGameKit VR, libGDX, ApertusVR, Torque3D, Urho3d, CopperCube and Skyline. Three of the most popular VR game engines from the list above are Unity, Unreal Engine and CryEngine.

For a complete beginner in VR gaming world the most suitable game engine from the enumeration above can definitely be Unity. Moving along this chapter we will discuss the benefits of using Unity game engine over the oth-

\section{Windows 10}

\section{hTсVIVE}

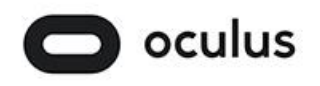

Microsoft HoloLens ers. First of all, the main programming language that Unity uses is C\#, a language that is easy to learn, versatile, object oriented and complex. When it comes to Unreal Engine or CryEngine, the programming language that is used for developing on these platforms is $\mathrm{C}++$ which is more sophisticated and harder to learn being especially used by professional developers who want a certain level of flexibility.

Alongside having a friendly user interface that is easy to use and explore, Unity also provides its users with high quality tutorials as well as a structured documentation which can be found easily on their website. In addition, for a beginner, finding assets for their projects can represent a difficult task, but this game engine has an integrated Asset Store in which the community can share assets that they create.

Another advantage of using Unity over another game engine is that it has the ability to compile the games for a large variety of platform such as: Oculus Rift, HTC Vive, Gear VR, Daydream, etc. In Figure 3 are presented all the platforms supported by Unity for virtual reality.

\section{\&B. Playstation.VR OpenVR Gear VR}

Fig. 3. Unity supported platforms (source: https://docs.unity3d.com/Manual/XR.html)

\section{A small glance into VR development}

Through this paper we will see how a small VR game can be created with ease by using the Unity game engine. The steps necessary for realizing a basic virtual reality $3 \mathrm{D}$ maze, in which the player is able to move forward, stop and turn left or right, are presented below. First things first, we will import the Google VR for Unity plugins into a blank Unity project. Then, we are going to delete the default 
camera object and replace it with GvrMain from the imported plugins. Moving along, we added a simple plane as our ground and a skybox which we took from the Asset Store (see Figure 4 and Figure 5).

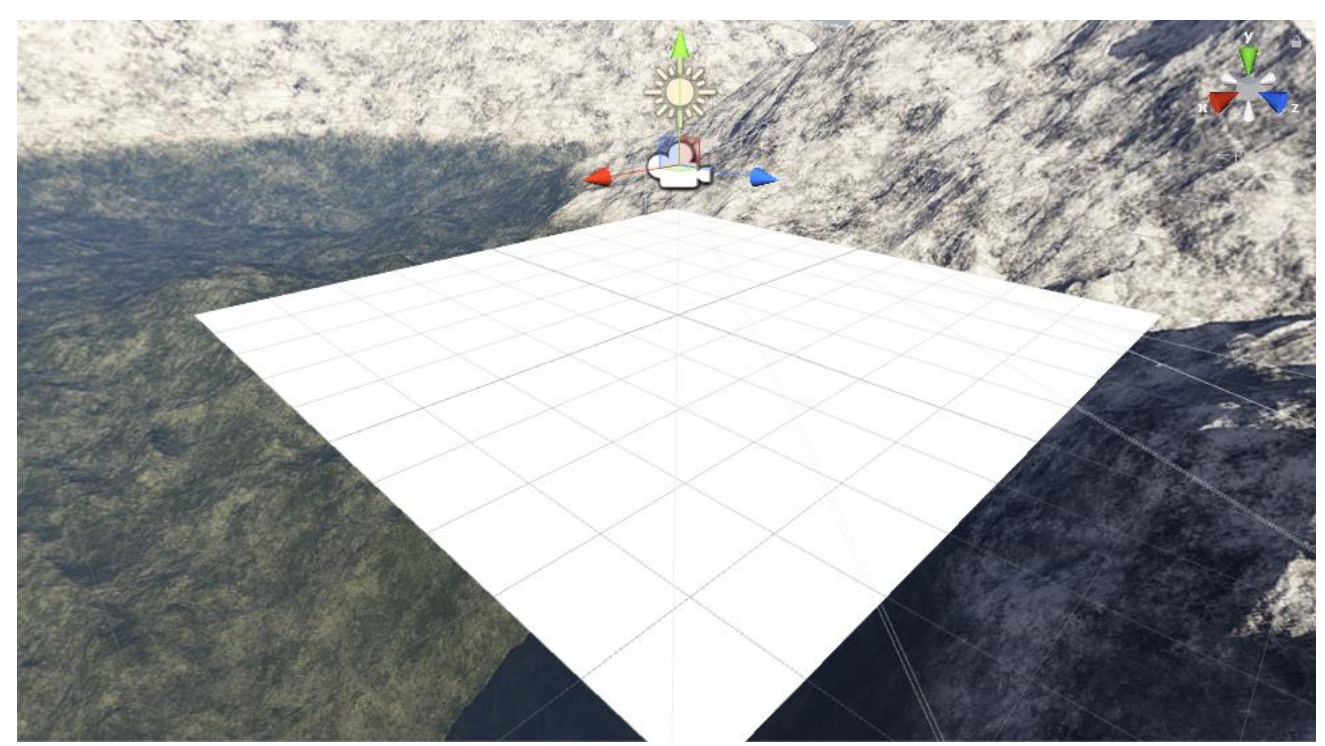

Fig. 4. Unity scene view (plane and skybox)

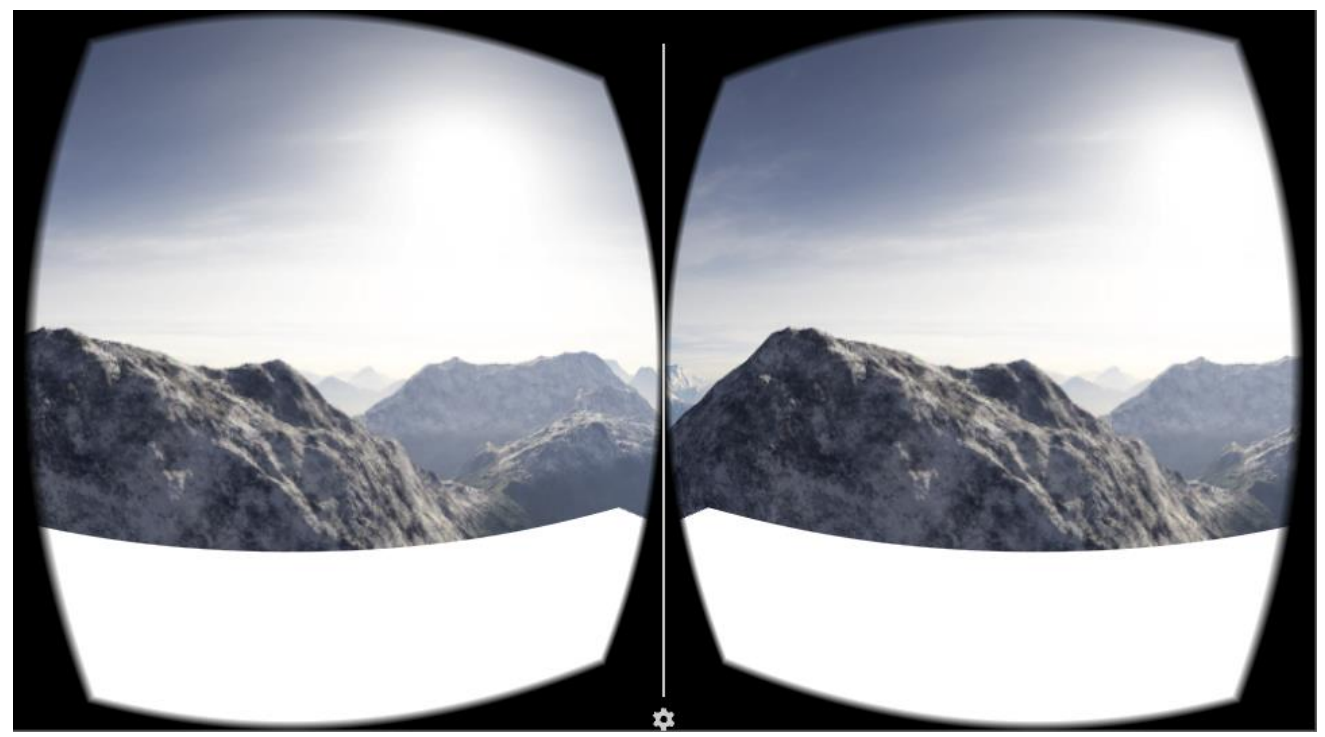

Fig. 5. Unity game view (plane and skybox)

For creating the maze, we downloaded a maze template from the Internet, we applied it on the plane (see Figure 6) and we started creating the walls (see Figure 7) using simple cubes by simply changing their dimensions according to the template. 


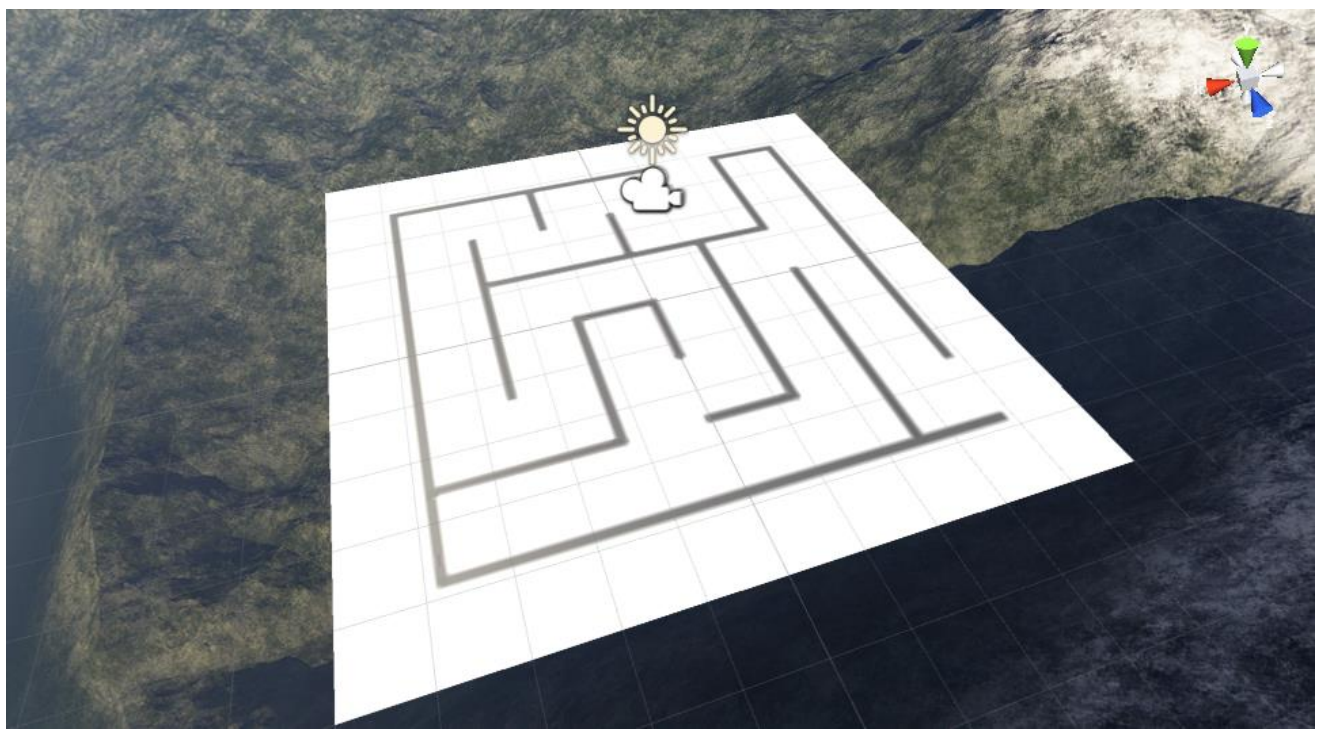

Fig. 6. Unity scene view (plane, skybox and maze template applied)

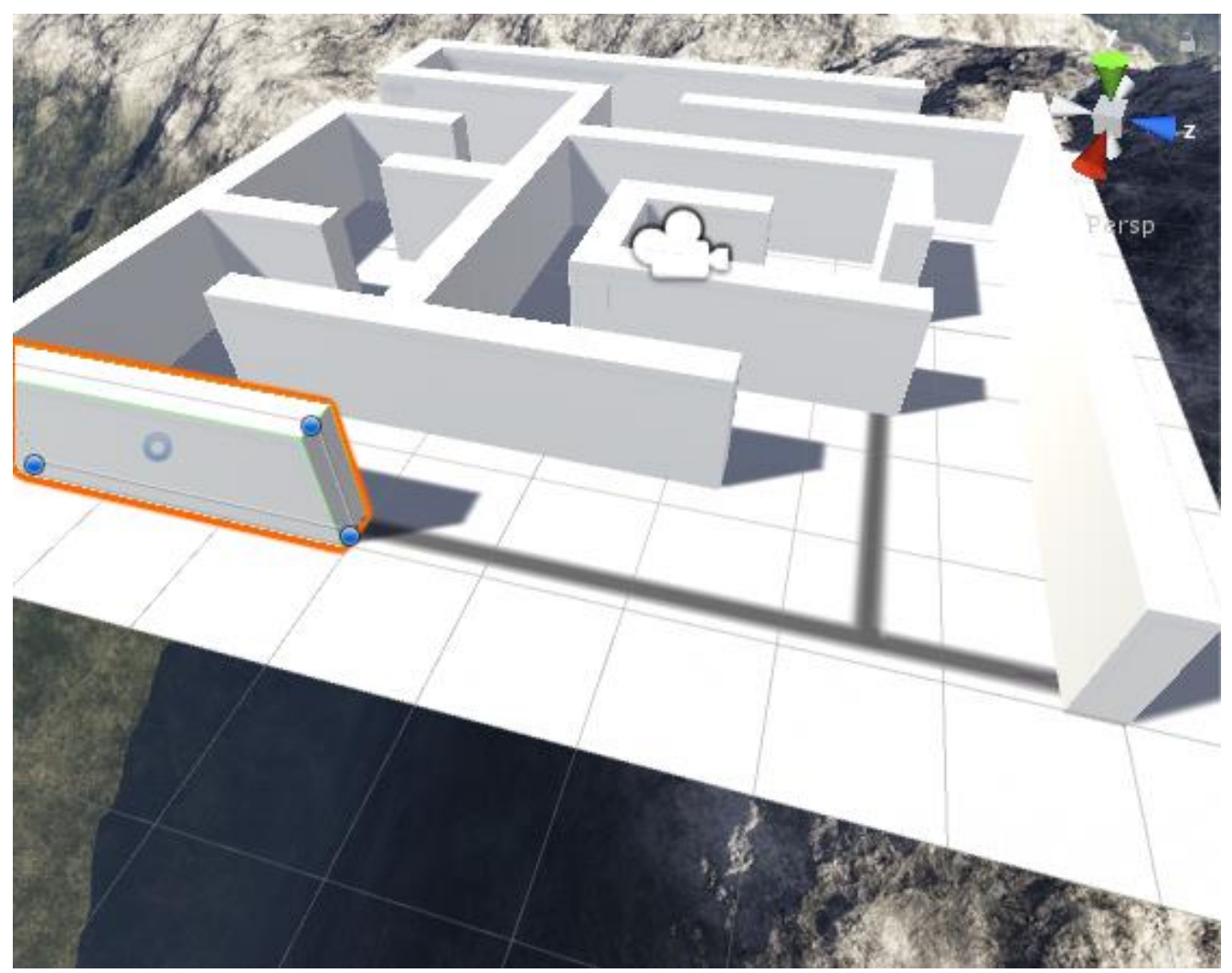

Fig. 7. Unity scene view (plane, skybox and maze template applied with constructed 3D walls)

At the same time, we downloaded a grass tex- walls and the plane (see Figure 8). ture from the Internet and we added it to the 


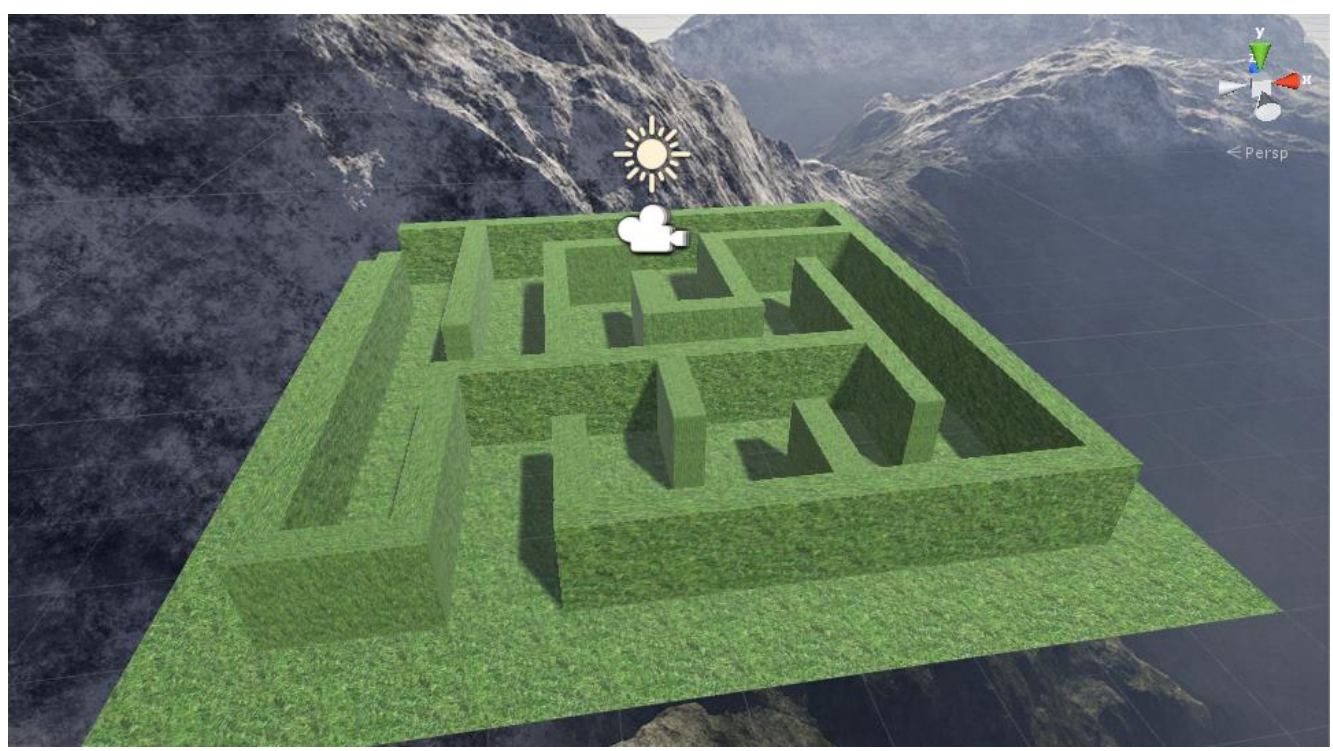

Fig. 8. Unity scene view (the final maze game scene)

Next up we added a "Rigidbody", a Capsule Collider and a C\# Script named Controller (see Figure 10) to our GvrMain object, so we make it behave like a character. In the added script we made the character walk automatically and stop if it looks down, and also, we store the staring position, so if the player finishes the maze he will be positioned at the starting point (see Figure 9). Figure 11 shows the final result of the project.

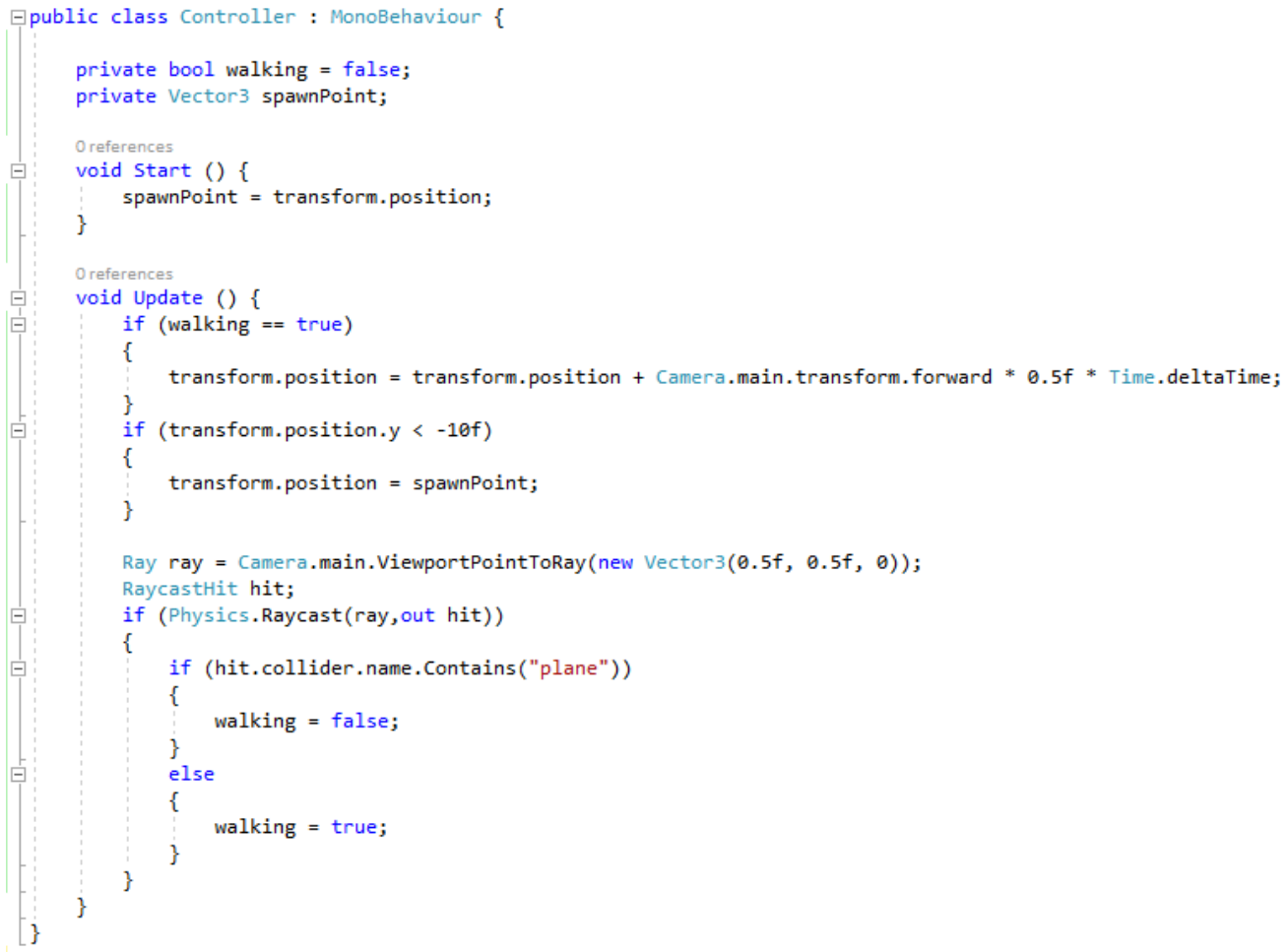

Fig. 9. C\# script for character movement and reset 


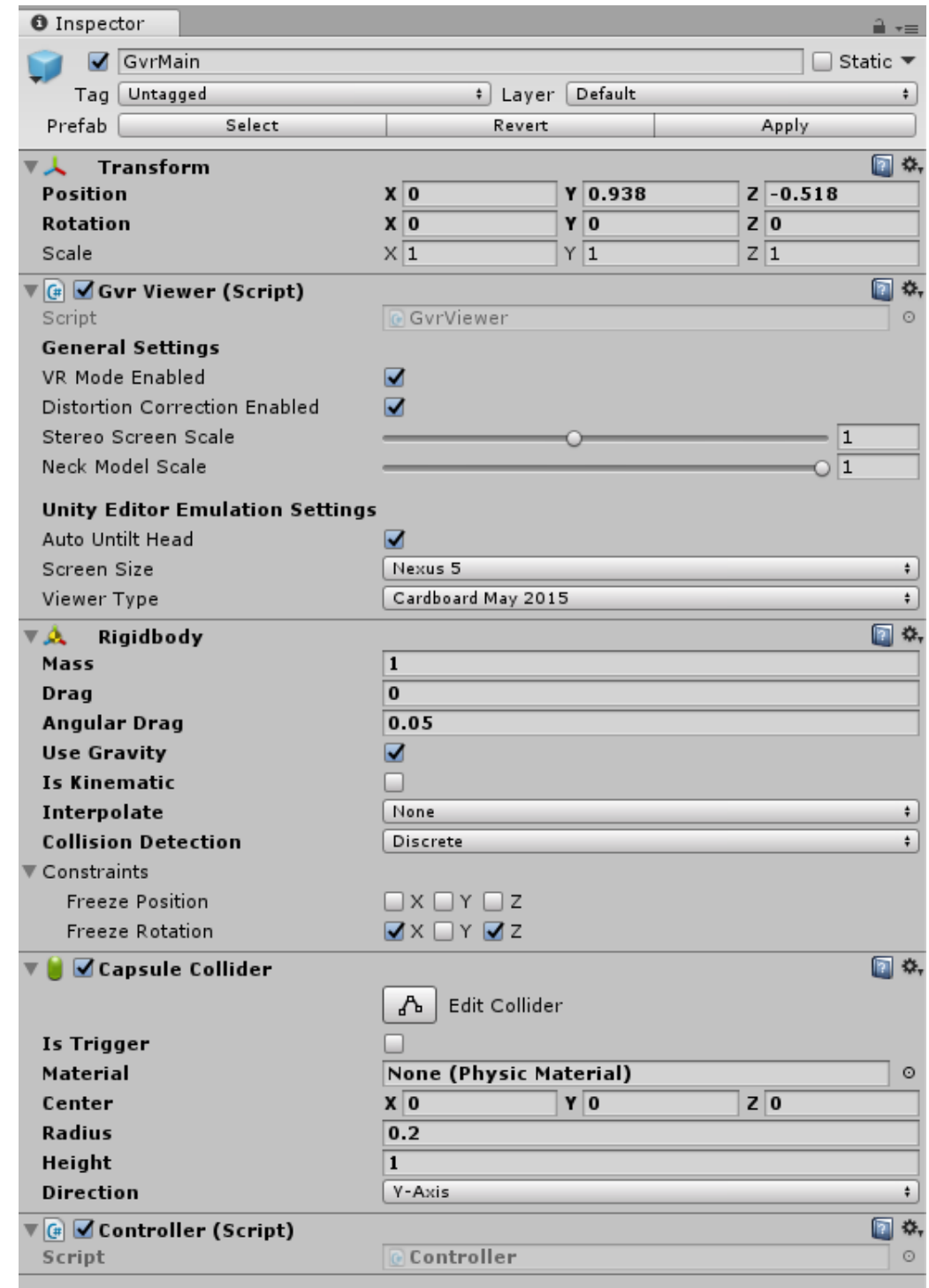

Fig. 10. The components added to the character in the Inspector Panel

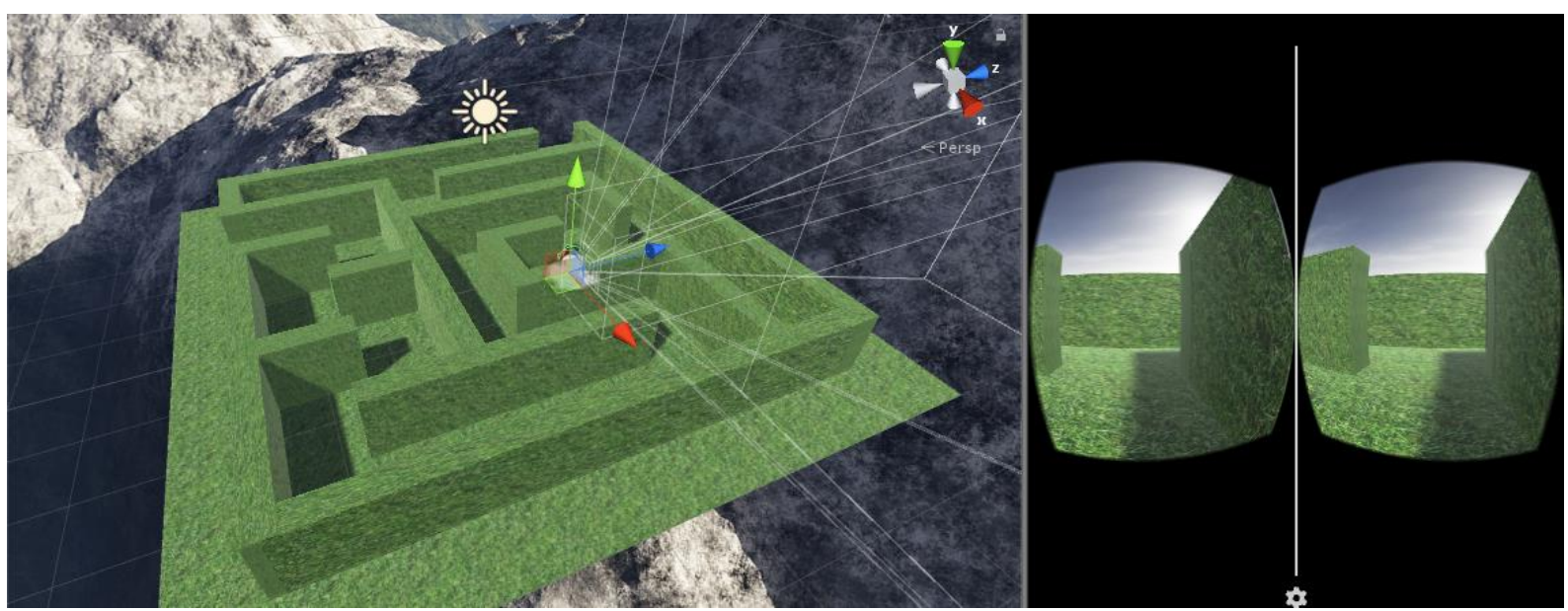

Fig. 11. Unity scene and game view of the final result

What has to be made from now is to export the game to one of the supported platforms.

\section{Conclusions}

In this paper, we conclude that virtual reality 
represents a powerful tool who can empower people by allowing them to create astonishing projects. Therefore, anyone who is willing to develop a virtual reality game can easily start by using the Unity game engine, because it is simple to use, learn and has all the documentation well organized on their website. At the same time, being so popular it has a lot of third party support from the entire community which makes it more accessible.

\section{References}

[1] C. Cruz-Neira, M. Fernandez and C. Portales, "Virtual Reality and Games," ResearchGate, May 2018. [Online]. Available: https://www.researchgate.net/publication/323418731_Virtual_Reality_and_Games

[2] P. Bouvier, F. Sorbier, P. Chaudeyrac and V. Biri, "Cross benefits between virtual reality and games," ResearchGate, May
2018. [Online]. Available: https://www.researchgate.net/publication/236260040_Cross_Benefits_Between_Virtual_Reality_And_Games

[3] N.R. Raajan and S. Gayathri, "Augmented Reality Based Virtual Reality," International Conference on Modeling, Optimization and Computing, Tamil Nadu, India, 2012, pp. 1559-1565.

[4] C. Woodford, "Virtual reality," May 2018. [Online]. Available: http://www.explainthatstuff.com/virtualreality.html

[5] Oculus.com, "Rift | Overview," Oculus, May 2018. [Online]. Available: https://www.oculus.com/rift/\#oui-csl-riftgames=star-trek

[6] Vive.com "Products | Vive," Vive, May 2018. [Online]. Available: https://www.vive.com/us/product/vivevirtual-reality-system

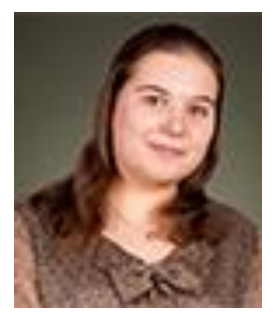

Cosmina ISAR has graduated The Faculty of Cybernetics, Statistics and Economic Informatics from The Bucharest University of Economic Studies in 2016. Currently she is studying for a Master degree in Economic Informatics at The Bucharest University of Economic Studies. Her current research focuses on game development using the Unity game engine. Other fields of interest include mobile devices, IoT and multimedia. 